\title{
Colonic content: effect of diet, meals, and defecation
}

\section{A. Bendezú ${ }^{1}$ \\ 21. Navazo ${ }^{2}$ \\ M. Mego ${ }^{1}$}

\author{
E. Monclus ${ }^{2}$
}

X. Merino ${ }^{3}$

A. Accarino $^{1}$

\author{
${ }^{1}$ Digestive System Research Unit, University \\ Hospital Vall d'Hebron, Centro de \\ Investigación Biomédica en Red de \\ Enfermedades Hepáticas y Digestivas \\ (Ciberehd), Universitat Autònoma de \\ Barcelona, Bellaterra (Cerdanyola del Vallès), \\ Spain \\ ${ }^{2}$ Department of Computer Languages and \\ System, Universidad Politécnica de Cataluña, \\ Barcelona, Spain \\ ${ }^{3}$ Radiology Department, University Hospital \\ 3 Vall d'Hebron, Barcelona, Spain
}

\section{Correspondence}

Fernando Azpiroz, MD, Digestive System Research Unit, Hospital General Vall d'Hebron, Barcelona, Spain.

Email: azpiroz.fernando@gmail.com

\begin{abstract}
Background: The metabolic activity of colonic microbiota is influenced by diet; however, the relationship between metabolism and colonic content is not known. Our aim was to determine the effect of meals, defecation, and diet on colonic content.

Methods: In 10 healthy subjects, two abdominal MRI scans were acquired during fasting, 1 week apart, and after 3 days on low- and high-residue diets, respectively. With each diet, daily fecal output and the number of daytime anal gas evacuations were measured. On the first study day, a second scan was acquired 4 hours after a test meal $(n=6)$ or after 4 hours with nil ingestion $(n=4)$. On the second study day, a scan was also acquired after a spontaneous bowel movement.

Results: On the low-residue diet, daily fecal volume averaged $145 \pm 15 \mathrm{~mL}$; subjects passed $10.6 \pm 1.6$ daytime anal gas evacuations and, by the third day, non-gaseous colonic content was $479 \pm 36 \mathrm{~mL}$. The high-residue diet increased the three parameters to $16.5 \pm 2.9$ anal gas evacuations, $223 \pm 19 \mathrm{~mL}$ fecal output, and $616 \pm 55 \mathrm{~mL}$ non-gaseous colonic content $(P<.05$ vs low-residue diet for all). On the low-residue diet, non-gaseous content in the right colon had increased by $41 \pm 11 \mathrm{~mL}, 4$ hours after the test meal, whereas no significant change was observed after 4-hour fast $(-15 \pm 8 \mathrm{~mL} ; P=.006$ vs fed). Defecation significantly reduced the non-gaseous content in distal colonic segments.

Conclusion \& Inferences: Colonic content exhibits physiologic variations with an approximate $1 / 3$ daily turnover produced by meals and defecation, superimposed over diet-related day-to-day variations.
\end{abstract}

\section{KEYWORDS}

colonic content, diet, fecal output, intestinal gas, meals

\section{1 | INTRODUCTION}

The human body hosts a symbiotic population of micro-organisms that accomplish key functions in host development and homeostasis. ${ }^{1}$ The largest population of microbiota is located within the digestive tract, specifically in the colon, which permits dynamic interactions across the mucosa. The upper part of the digestive tract has primarily a nutritional function: useful components, such as nutrients, water, and some minerals, are extracted from the ingested material by a process of digestion and absorption. Non-absorbed residues left over pass into the colon and are metabolized by microbiota with symbiotic benefit. Hence, the colon, located at the end of the digestive tract, rather than being a simple fecal-producing conduit, behaves as a specialized marsupial pouch and a complex metabolic organ.

Microbiota metabolism of meal residues releases a series of metabolites that in turn serve as substrates for other subsets of microbiota. Hence, the colon contains an active mass of living matter, formed by microbiota, meal residues, and secondary metabolic products in a dynamic chain of metabolic reactions. Based on this definition, we propose the concept of colonic content as a biomass where 
microbiota adapts to its environment depending on the primary residues from the diet and as it changes along the colon. Furthermore, dietary habits influence microbiota composition: while fiber-rich diets increase its diversity, diets low in fermentable residues, such as the low-FODMAPs diet, induce the opposite effect. ${ }^{2-4}$ However, the impact of these functional effects on the physical characteristics of colonic content is not known.

Our aim was to determine the effect of meals, defecation, and diet on colonic content volume. Abdominal CT imaging is useful for detecting variations in colonic content in relation to physiologic stimuli, i.e. meal ingestion in healthy subjects, as well as in relation to symptoms, particularly in patients complaining of abdominal bloating and distension. ${ }^{5,6}$ However, the radiation involved in CT limits its application for repeat imaging. Hence, in this study, we applied an image analysis method based on abdominal MRI scans.

\section{2 | MATERIALS AND METHODS}

\section{1 | Participants}

Eighteen healthy subjects without gastrointestinal symptoms (11 women, 7 men; age range: $24-55$ years) participated in the study; 11 participated in more than one experiment (see "Experimental design"). Antibiotic consumption during the previous 2 months was an exclusion criterion. Prior to inclusion in the study, healthy subjects were instructed to complete a clinical questionnaire to confirm the absence of gastrointestinal symptoms; all reported normal bowel habits $(7.0 \pm 0.1$ bowel movements per week) and stool form (3.9 \pm 0.2 score on the Bristol scale). ${ }^{7}$ Subjects gave their written informed consent to participate in the study. The study protocol was previously approved by the Institutional Review Board of the University Hospital Vall d'Hebron.

\section{2 | Experimental design}

\subsection{1 | Effect of diet}

Subjects ( $n=10$ ) were instructed to follow a 3-day low-residue diet and a 3-day high-residue diet with a 3-day diet-free interval (see Procedures). During the 3 days on the low- and high-residue diets, they were instructed to complete daily clinical questionnaires, measure fecal output, and count the number of daytime anal gas evacuations; on the morning immediately after each period, an abdominal MRI scan was acquired (in all but one participant) after an overnight fast to measure colonic content (see Procedures).

\subsection{2 | Effect of meal ingestion}

The day after the low-residue diet period, an abdominal MRI scan was acquired to measure colonic content after an overnight fast; thereafter, a second scan was acquired 4 hours after a test meal $(n=6)$ or after a further 4-hour fast (with nil ingestion; $n=4$ ). The test meal consisted of $250 \mathrm{~g}$ of white beans and one banana (100 g) with a total content of $54.0 \mathrm{~g}$ fructose, $7.4 \mathrm{~g}$ glucose, $2.9 \mathrm{~g}$ sorbitol, $2.7 \mathrm{~g}$

\section{Key points}

- The metabolic activity of colonic microbiota is influenced by meals and diet; our aim was to determine the effect of meals, defecation, and diet on colonic content.

- Our study identified marked physiologic variations in colonic content combining different cycles: variations produced by meals and defecation, superimposed over day-to-day variations related to changes in diet.

- Our data on the fractional clearance rate suggest a very active turnover of colonic content, which might permit potentially rapid adaptation of microbiota to changes in the intraluminal environment.

mannitol, and $16.1 \mathrm{~g}$ fiber (including $1.5 \mathrm{~g}$ galacto-oligosaccharides and $1.4 \mathrm{~g}$ fructo-oligosaccharides)., ${ }^{8,9}$

\subsection{3 | Effect of defecation}

The day after the high-residue diet period, an abdominal MRI scan was acquired to measure colonic content after an overnight fast; thereafter, subjects $(n=15)$ remained in the laboratory without ingesting anything until they had a spontaneous bowel movement; fecal output (stool weight and volume) was then measured (see procedure in "Measurement of fecal output") and a second scan was acquired.

\subsection{4 | Validation studies}

\section{Gas infusion}

In 10 subjects, a balloon catheter was introduced into the rectum and fixed by inflating the balloon with 5-mL air. MRI scans were acquired immediately before and after the intrarectal infusion of a known volume of air (range: 50-170 mL; Fig. 4). Scans were analyzed blindly to measure the changes in intracolonic volume.

\section{Inter-observer variability}

A series of 20 scans was independently and blindly analyzed by two investigators ( $A B$ and $M M$ ).

\section{3 | Procedures}

\subsection{1 | Dietary interventions}

Participants were given detailed menus with the specific foodstuffs and their amounts in every meal during the 3-day diet period; foodstuffs were available at the clinical study unit and were offered to the participants to take home. The menus additionally included white bread, cheese, and ham as optional side courses or snacks should the participants feel hungry. Strict adherence to the diet was requested, and participants were asked how closely they had complied with the diet when they reported to the hospital the day after each period. 
During the low-residue diet period (8 $\mathrm{g}$ fiber per day), the diet was restricted to the following foodstuffs: a) meat, fish, fowl, and eggs; b) broth, lettuce, tomatoes, avocado, and olives; c) rice, pasta, bread, and cereals; d) dairy products; e) pears, apples, tangerines, and oranges; and f) sugar, chocolate, tea, coffee, wine, vinegar, and oil.

During the high-residue diet period (35-g fiber per day), the diet included in each meal (breakfast, lunch, and dinner) at least one portion of the following: (i) bread, cereals, and pastries made of whole wheat or corn; (ii) white beans, mixed vegetables, or chickpeas; (iii) pumpkin puree, vegetable puree, or spinach puree; and (iv) bananas, figs, peaches, grapes, and prunes.

\subsection{2 | Clinical questionnaires}

Participants were instructed to complete a questionnaire at the end of each day of each diet period. The questionnaire evaluated the following parameters: (i) subjective sensations of flatulence (defined as anal gas evacuation), abdominal bloating (pressure/fullness), sensation of abdominal distension (girth increment), borborygmi, and abdominal discomfort/pain by corresponding 0-10 analog scales; and (ii) digestive well-being and mood using a 10-point scale graded from +5 (excellent) to -5 (very bad); number of bowel movements and stool form by the Bristol scale. ${ }^{7}$

\subsection{3 | Fecal output measurement}

During the 3 days on each diet, participants were instructed to defecate in a disposable pan to measure the weight of each stool using a digital weighing scale (BT-32013; El Corte Ingles, Madrid, Spain) and then stool volume by immersing the stool in water within a graded beaker and recording the water level change. The weighing scale, disposable pans, and beaker were provided by the investigator.

\subsection{4 | Number of anal gas evacuations}

During the 3 days on each diet, the number of anal gas evacuations was measured using an event marker (DT2000 Memory Stopwatch; Digi sport instruments, Shanggiu, China). Participants were instructed to carry the event marker during the day and register every passage of anal gas. This method had been used previously with reproducible and consistent results ${ }^{10,11}$; furthermore, studies measuring the number of gas evacuations simultaneously by an event marker and continuous recording of anal gas evacuation showed a very good correlation. ${ }^{12-15}$

\subsubsection{Colonic content measurement}

All measurements, except those in the post-defecation study, were taken in the morning before a bowel movement. MR imaging examinations of the colon were performed using a 1.5-T MR imaging system (Aera; Siemens Healthcare, Erlangen, Germany) with 2 six-channel phased-array abdominal coils to cover the whole abdomen. The abdomen was imaged obtaining a T2-weighted HASTE sequence in the coronal plane (1400-ms repetition time, $90-\mathrm{ms}$ echo time, $3.5-\mathrm{mm}$ slice thickness, $180^{\circ}$ flip angle, and $256 \times 256$ matrix resolution) during two apneas of around 20 seconds each and a T1-weighted VIBE Fat-Sat sequence in the coronal plane (3.71-ms repetition time, $1.66-\mathrm{ms}$ echo time, $1.5-\mathrm{mm}$ slice thickness, $10^{\circ} \mathrm{flip}$ angle, and $320 \times 189$ matrix resolution) in one apnea of 12 seconds. No drugs or contrast were used.

\section{4 | Data analysis}

Analysis of the images was made using an original software developed for this purpose. The program allows semiautomatic segmentation of the colon on the images using a region-growing-based algorithm. First, an anisotropic contrast enhancement filter is applied to enhance the boundary of the colon without loss of inside detail. Then, seed points are placed which expand depending on the gray-level mapping defined by the window-level setting of the images. To facilitate colonic segmentation, a toolkit was developed that permits enlarging or reducing the segmentation obtained by the region-growing algorithm. Colonic segmentation was correlated in T2 and T1 images. Non-gaseous colonic content was measured in T1 colonic images, and gaseous colonic content was measured by subtracting T1 from T2 colonic volumes. A three-dimensional reconstruction program with $360^{\circ}$ rotation over the three dimensions, previously developed for morphovolumetric analysis of CT images, was adapted for MRI analysis to facilitate measurement of volumes in selected regions of the colon. ${ }^{6}$ The different blocks of the analysis program underwent a series of interim validation steps during the development phase. In the validation studies (see below), images before and after colonic infusion of known volumes of air, as well as before and after defecation, were compared. After inter-observer reproducibility of results was proved (see validation studies), images were codified and analyzed by one investigator ( $\mathrm{AB}$ or $\mathrm{MM}$ ) blinded to the source, acquisition, and any preceding intervention. In some images, the small and large intestine (particularly the cecum, transverse, and sigmoid colon) overlapped, and an experienced radiologist (XM) provided expert support.

\section{5 | Statistical analysis}

Means $( \pm S E)$ of the variables measured were calculated. The Kolmogorov-Smirnov test was used to check the normality of data distribution. Parametric normally distributed data were compared by Student's t-test for paired or unpaired data; otherwise, the Wilcoxon signed-rank test was used for paired data and the Mann-Whitney $U$ test for unpaired data. The reproducibility of measurements in the validation studies was analyzed using Bland Altman plots. ${ }^{16,17}$

\section{3 | RESULTS}

\subsection{Effect of diet}

On reporting to the laboratory for abdominal imaging the morning after each diet period, all participants confirmed their adherence to diets, with no major deviations from the menus provided. 
TAB LE 1 Effects of diet on colonic content indices and sensations

\begin{tabular}{|c|c|c|c|c|}
\hline & Low & High & $\Delta, \%$ & $P$ \\
\hline $\begin{array}{l}\text { Daily fecal } \\
\text { output, g }\end{array}$ & $131 \pm 15$ & $211 \pm 20$ & $70 \pm 19$ & .004 \\
\hline $\begin{array}{l}\text { Daily fecal } \\
\text { output, mL }\end{array}$ & $145 \pm 15$ & $223 \pm 19$ & $65 \pm 20$ & .006 \\
\hline $\begin{array}{l}\text { Stool density, } \\
\mathrm{g} / \mathrm{mL}\end{array}$ & $0.91 \pm 0.03$ & $0.93 \pm 0.01$ & $3 \pm 4$ & .662 \\
\hline Bristol score & $4.5 \pm 0.1$ & $4.3 \pm 0.2$ & $-3 \pm 4$ & .227 \\
\hline $\begin{array}{l}\text { Bowel } \\
\text { movements, } \\
\text { No/3 day }\end{array}$ & $3.6 \pm 0.4$ & $4.0 \pm 0.4$ & $17 \pm 12$ & .469 \\
\hline $\begin{array}{l}\text { Anal gas } \\
\text { evacuations, } \\
\text { No/1 day }\end{array}$ & $10.6 \pm 1.6$ & $16.5 \pm 2.9$ & $60 \pm 13$ & .010 \\
\hline $\begin{array}{l}\text { Non-gaseous } \\
\text { colon content }\end{array}$ & $479 \pm 36$ & $616 \pm 55$ & $35 \pm 16$ & .038 \\
\hline $\begin{array}{l}\text { Gaseous colon } \\
\text { content, } \mathrm{mL}\end{array}$ & $162 \pm 19$ & $208 \pm 445$ & $49 \pm 39$ & .196 \\
\hline $\begin{array}{r}\text { Daily content } \\
\text { clearance, \% }\end{array}$ & $34 \pm 5$ & $38 \pm 5$ & $4 \pm 6$ & .276 \\
\hline $\begin{array}{l}\text { Flatulence, } \\
\text { score }\end{array}$ & $1.8 \pm 0.6$ & $2.8 \pm 0.5$ & $43 \pm 21$ & .238 \\
\hline Pain, score & $0.3 \pm 0.2$ & $0.7 \pm 0.4$ & $6 \pm 6$ & .388 \\
\hline $\begin{array}{l}\text { Distension, } \\
\text { score }\end{array}$ & $0.3 \pm 0.3$ & $0.9 \pm 0.4$ & $3 \pm 3$ & .282 \\
\hline $\begin{array}{l}\text { Borborygmi, } \\
\text { score }\end{array}$ & $1.7 \pm 0.7$ & $2.6 \pm 0.6$ & $72 \pm 61$ & .343 \\
\hline $\begin{array}{l}\text { Bloating, } \\
\text { scores }\end{array}$ & $0.4 \pm 0.3$ & $0.8 \pm 0.4$ & $4 \pm 4$ & .454 \\
\hline $\begin{array}{l}\text { Well-being } \\
\text { score }\end{array}$ & $3.9 \pm 0.3$ & $3.7 \pm 0.4$ & $-10 \pm 6$ & .531 \\
\hline مصpd, score & $4.1 \pm 0.2$ & $4.1 \pm 0.3$ & $-1 \pm 2$ & .923 \\
\hline
\end{tabular}

On the low-residue diet, daily fecal output averaged $145 \pm 15 \mathrm{~mL}$ (Table 1), subjects passed 10.6 \pm 1.6 anal gas evacuations during the day and, by the third day, non-gaseous colonic content was $479 \pm 36 \mathrm{~mL}$ (Fig. 1). Based on average daily fecal output and on the volume of intracolonic non-gaseous content, $34 \% \pm 5 \%$ of colonic content was cleared every day. Non-gaseous and gaseous content was uniformly distributed along the colon (Fig. 1).

The high-residue diet raised the number of anal gas evacuations (by $60 \% \pm 13 \%$ ) and fecal output (by $65 \% \pm 20 \%$ ), with no changes in stool density, consistency, and frequency (Table 1). Non-gaseous content increased (Figs 1 and 2). Daily colonic content daily clearance was $38 \% \pm 5 \%$. Gas content in the colon tended to increase, but the differences were not statistically significant (Table 1 and Fig. 2). Participants reported low-score abdominal symptoms on both diets.

\subsection{Effect of test meal}

In the subjects who consumed the test meal after the fasting scan and had a second scan 4 hours later $(n=6)$, non-gaseous
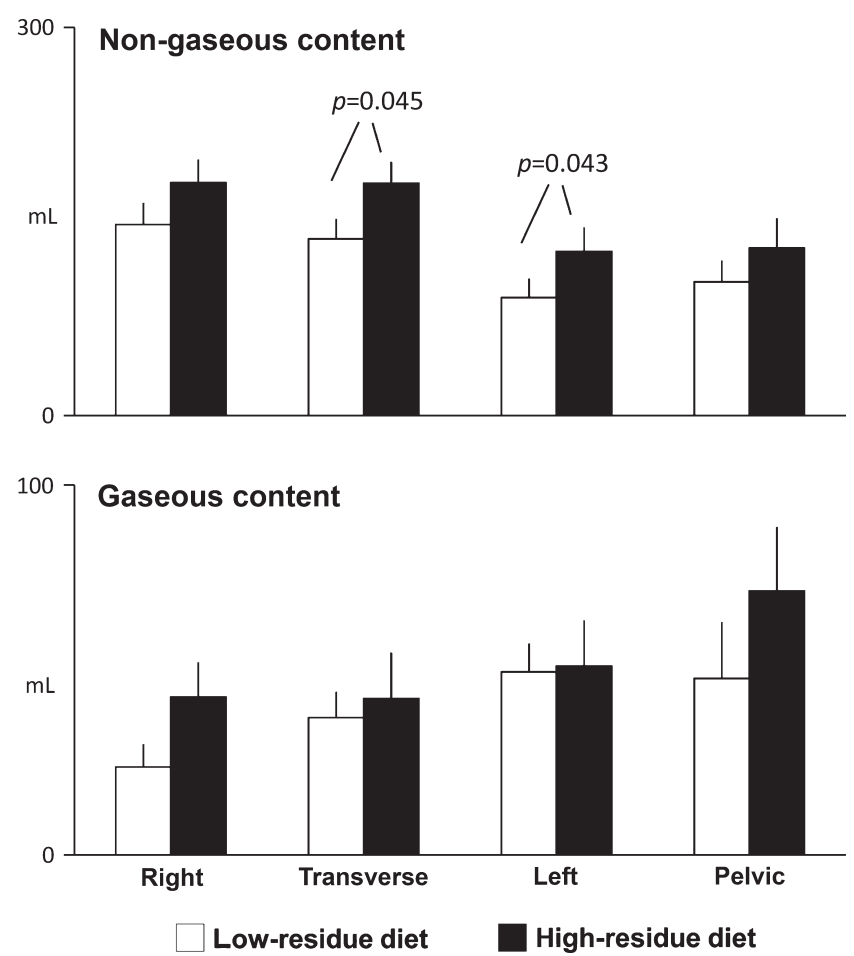

FIGURE 1 Effect of diet on colonic content. Overnight fast measurements after 3-day low- and high-residue diets $(n=9)$. The high-residue diet was associated with a significant increase in total volume of non-gaseous content, but not of gas

colonic content in the proximal colon significantly increased (by $37 \% \pm 15 \% ; P=.007$ ) (Fig. 3). Overall gas content tended to increase (by $31 \% \pm 14 \% ; P=.064$ ) (Fig. 3 ). The subjects who continued fasting after the first scan and had a second scan 4 hours later $(n=4)$ exhibited a non-gaseous mass reduction in the pelvic colon (by $-29 \% \pm 11 \% ; P=.040$ vs first scan) (Fig. 3). When the changes produced by meal ingestion vs the variations observed during prolonged fasting were compared, only the increase in non-gaseous content in the right colon was statistically significant $(P=.006)$.

\section{3 | Effect of defecation}

An interval of $102 \pm 10$ minutes elapsed between the basal scan and the scan taken immediately after a spontaneous bowel movement. Defecation reduced intracolonic volume of non-gaseous content in all colonic segments; however, the change was more pronounced in the pelvic colon (Fig. 4). The volume of fecal mass evacuated corresponded to $38 \% \pm 5 \%$ of non-gaseous colonic content, which corresponds to the colonic clearance rate. After defecation, gas volume in the pelvic colon also decreased $(P<.001)$.

\section{4 | Validation studies}

Overall, the decrease in non-gaseous colonic content $(140 \pm 23 \mathrm{~mL})$ was similar to the volume of stool evacuated (136 $\pm 20 \mathrm{~mL}$ ) (Fig. 5A), and conversely, the increase in colonic content after rectal gas infusion 
FIGURE 2 Examples of non-gaseous colonic content in the same subject on lowand high-residue diets after overnight fast

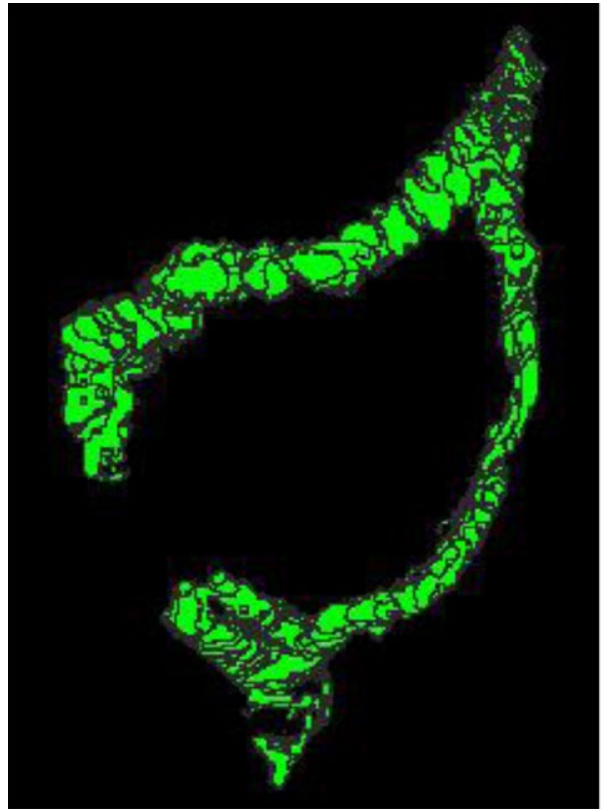

Low-residue diet

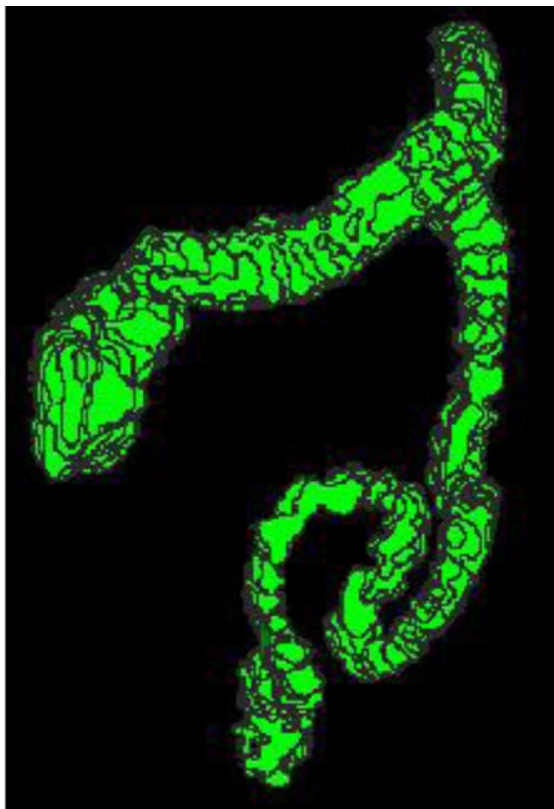

High-residue diet
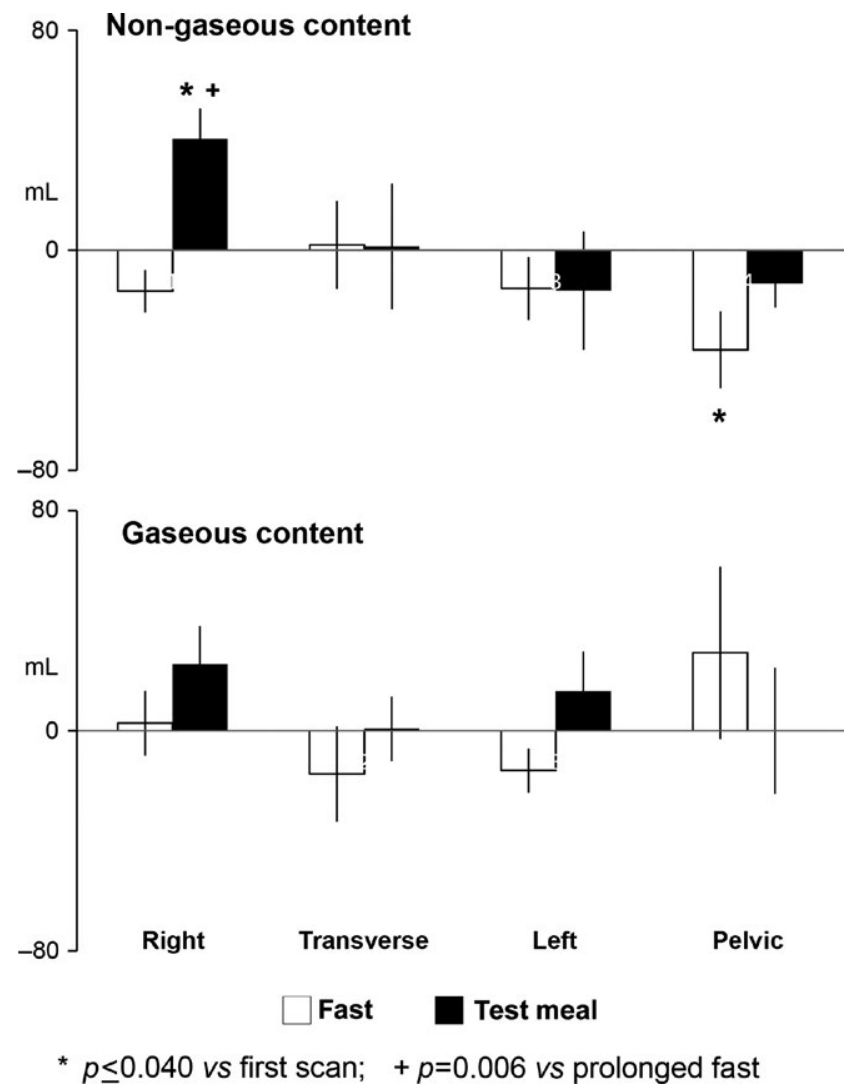

FIGURE 3 Effect of test meal on colonic content. Changes in colonic content for $4 \mathrm{~h}$ after continued overnight fasting $(\mathrm{n}=4)$ and $4 \mathrm{~h}$ after the test meal $(n=6)$

$(117 \pm 12 \mathrm{~mL}$ ) was similar to the volume of gas infused (126 $\pm 12 \mathrm{~mL})$ (Fig. 5B). Independent measurements of colonic content by two observers (Fig. 5C) yielded similar results for the total colon $(701 \pm 40$ and $702 \pm 37 \mathrm{~mL}$, respectively) and for individual regions (188 \pm 11

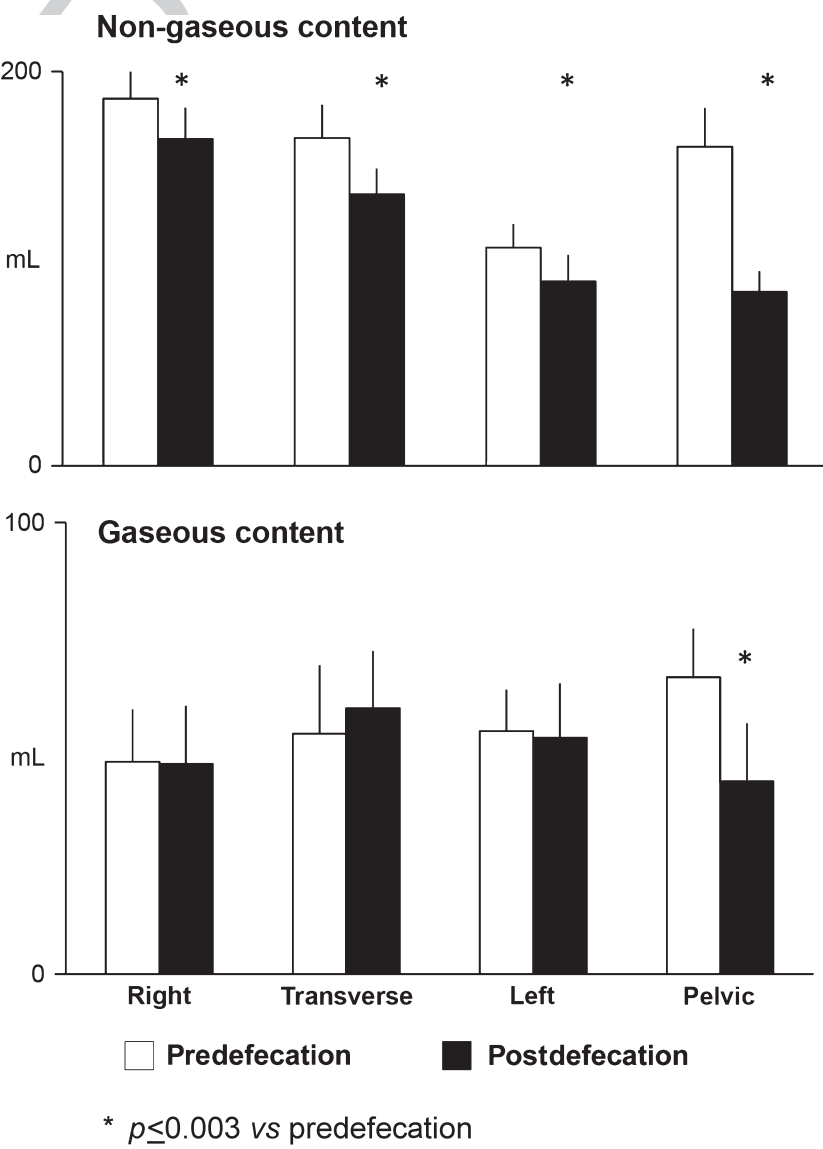

FIGURE 4 Effect of defecation on colonic content. Overnight fast measurements $102 \pm 10$ min before and immediately after a spontaneous bowel movement

and $199 \pm 13 \mathrm{~mL}$ right colon, $199 \pm 11$ and $199 \pm 11 \mathrm{~mL}$ transverse colon, $157 \pm 12$ and $157 \pm 10 \mathrm{~mL}$ left colon, $157 \pm 18$ and $147 \pm 16 \mathrm{~mL}$ pelvic colon). 


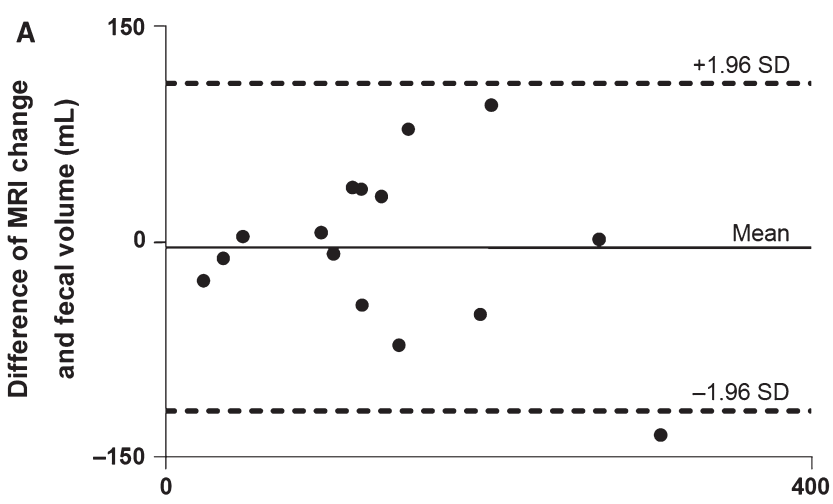

Average of MRI change and fecal volume $(\mathrm{mL})$

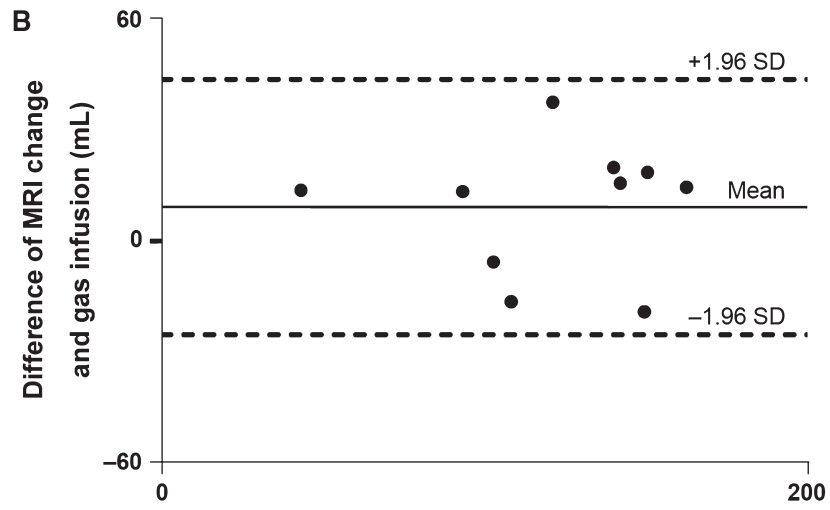

Average of MRI change and gas infusion $(\mathrm{mL})$

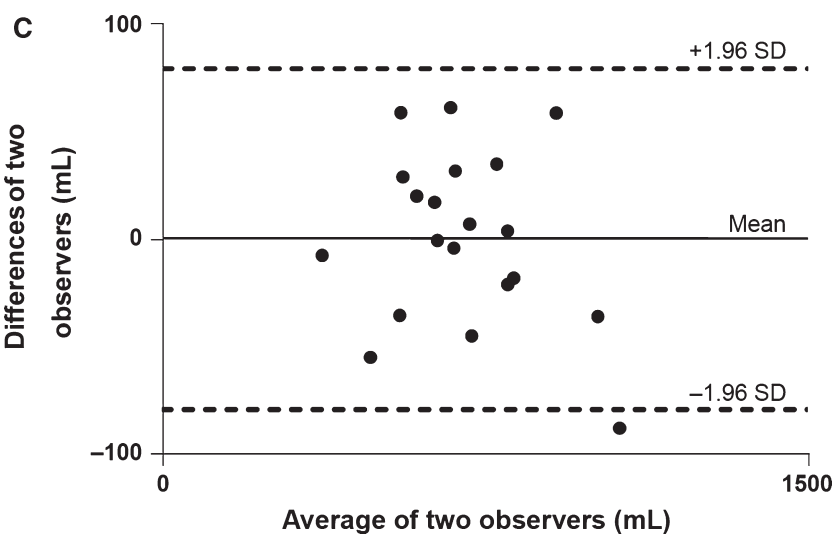

FIGURE 5 Validation studies. Bland Altman plots of colonic content measured: (A) After defecation (volume change vs stool volume); (B) after colonic gas infusion (volume change vs gas infused); and (C) by two investigators independently

\section{4 | DISCUSSION}

Our study provides original and direct experimental evidence on the fractional clearance rate of colonic content, around a one-third daily change in healthy subjects. These data suggest that the turnover of colonic content may be very rapid and occur in a matter of days. Furthermore, our study shows a marked influence of diet on

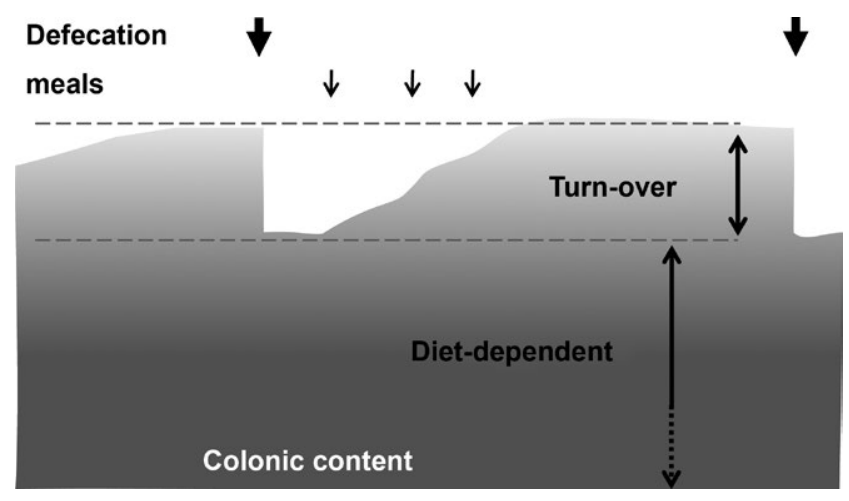

FIGURE 6 Schematic representation of results. Physiological variations in colonic content: cyclic turnover produced by meals and defecation, superimposed to day-to-day variations related to diet

colonic content, accounting for approximately $30 \%$ variations of volume (Fig. 6).

As in previous studies, intraluminal content was evenly distributed along the colon, ${ }^{6}$ and the distribution was not affected by diet. However, the mass of non-gaseous colonic content was influenced by the amount of non-absorbable residues in the diet; the change was considerable and rapid, and already significant (by over 30\%) after a 3-day intervention period. Exactly to what extent the increase in content is related to the presence of meal residues, metabolic products or microbiota proliferation is not known; however, given the chain of reactions in colonic content, all conceivably contributed.

Diet also influenced fecal output, which increased in parallel to nongaseous colonic content; however, stool density, form, or frequency were not affected. Over one-third of colonic content was cleared daily and replaced, indicating that this is a very dynamic process. The fractional clearance rate of colonic content was not influenced by the diet or colonic content volume. Comparison of colonic content before and after defecation indicates that the process of defecation involves not only rectal emptying but also a major redistribution of contents along the colon, which conceivably takes place in the prodromic phase preceding fecal evacuation. Of note, intracolonic volumes of non-gaseous content were in the range of those previously reported using both CT and MRI imaging., 6,16,18,19

Individual meal ingestion also influenced colonic content. Indeed, 4 hours after a relatively high-residue meal, non-gaseous content in the right colon increased, possibly due to the arrival of undigested/ unabsorbed residues from the small bowel, while no consistent changes in the rest of the colon were observed. In a previous study, we measured intraluminal volume changes by $\mathrm{CT}$ at an earlier phase of the postprandial period, conceivably before the arrival of residues from the small bowel ( 90 minutes after the meal). At this interval, only a redistribution of contents was observed, with a mass movement from the descending to the pelvic colon, which could be related to gastrocolic reflexes released by meal ingestion. ${ }^{6}$ Interestingly, prolonged fasting in this study was associated with a volume reduction in the distal colon, which could be related to substrate consumption and/or to water absorption with consolidation of the fecal bolus.

In contrast to the physiologic differences in the bulk of colonic content associated with the single test meal and the dietary intervention, 
gas content in the colon remained fairly steady: gas volume tended to increase after the test meal and on the high flatulogenic diet; however, the changes were not statistically significant. A body of experimental evidence indicates that intestinal gas is produced in the fermentation process residues by colonic microbiota, and the volume of gas produced depends primarily on the amount of ingested fermentable residues reaching the colon. ${ }^{10,20-24}$ However, despite the importance of meals and diet on intestinal gas production demonstrated by these previous studies, our current data show their impact on intracolonic gas content to be limited, conceivably because intestinal gas homeostasis is a highly dynamic and efficient process. A large part of the gas produced is rapidly absorbed through the gut-blood barrier and eliminated via breath, and another fraction is metabolized by gas-consuming pools of gut microbiota. ${ }^{21,22,25,26}$ On top of this production/elimination balance, the colon actively moves and clears gas out of the gut via the anus, so that the content of gas within the colon is kept fairly constant. ${ }^{14,27-30}$ As in previous studies, dietary manipulations influenced the number of anal gas evacuations which reflects an increase in the volume of gas evacuated via the anus. ${ }^{10,31}$ In a previous study based on abdominal CT imaging, we observed an increase in intestinal gas volume in the early postprandial period, probably related to passage of ileal gas into the colon, ${ }^{5}$ and our current data show that, by 4 hours, the gas volume had returned to fasting level.

We wish to acknowledge several limitations and potential pitfalls of our study and the attempts made to minimize their potential impact. No direct measurements of the effect of diet on transit time were taken, although no differences in stool frequency and consistency on the Bristol scale, indicators of transit, ${ }^{7}$ were detected. Participants were unblinded to the dietary interventions, which may have influenced perceived symptoms and the number of gas evacuations, although they were not aware of the expected effects. We strived to control diet adherence by providing detailed menus and confirming compliance at the end of each 3-day period; however, consumption diaries were not used. Dietary supplementation did not influence digestive symptoms but did affect colonic volumes measured by MRI. Part of the software used to measure colonic volumes was originally developed for CT analysis; its application for MRI analysis has been partly validated but other steps remain.

Our data demonstrate the influence of dietary residues and fecal evacuation on the bulk of colonic content and provide some insight that may aid understanding of the biological cycle of colonic content and its potential adaptation to variations in the intraluminal environment.

\section{ACKNOWLEDGMENTS}

The authors thank Christine O'Hara for English editing and Gloria Santaliestra for secretarial assistance.

\section{FUNDING}

4 This study was supported by the Spanish Ministry of Economy and Competitiveness (Dirección General de Investigación Científica y Técnica, SAF 2013-43677-R); Ciberehd is funded by the Instituto de Salud Carlos III.

\section{DISCLOSURE}

No competing interests declared.

\section{AUTHOR CONTRIBUTION}

$A B$ and $M M$ contributed equally as first authors; $A B$ and $M M$ did study management, conducted experiments, and analyzed data; EM developed MRI analysis program; XM supervised MRI acquisition and analysis; AA supervised studies; JRM interpreted data and revised manuscript; IN developed MRI analysis program; FA designed study, interpreted data, and prepared manuscript.

\section{REFERENCES}

1. Simren M, Barbara G, Flint $\mathrm{HJ}$, et al. Intestinal microbiota in functional bowel disorders: a Rome foundation report. Gut. Jan 2013;62:159176.

2. Albenberg LG, Wu GD. Diet and the intestinal microbiome: associations, functions, and implications for health and disease. Gastroenterology. May 2014;146:1564-1572.

3. David LA, Maurice CF, Carmody RN, et al. Diet rapidly and reproducibility alters the human gut microbiome. Nature. 2014;505:559-573.

4. Halmos EP, Christophersen CT, Bird AR, Shepherd SJ, Gibson PR, Muir JG. Diets that differ in their FODMAP content alter the colonic luminal microenvironment. Gut. Jan 2015;64:93-100.

5. Bendezú A, Barba E, Burri E, et al. Intestinal gas content and distribution in health and in patients with functional gut symptoms. Neurogastroenterol Motil. 2015;27:1249-1257.

6. Bendezu RA, Barba E, Burri E, et al. Colonic content in health and its relation to functional gut symptoms. Neurogastroenterol Motil. 2016; doi:10.1111/nmo.12782 [Epub ahead of print] online.

7. Lewis SJ, Heaton KW. Stool form scale as a useful guide to intestinal transit time. Scand J Gastroenterol. 1997;32:920-924.

8. Muir JG, Rose R, Rosella O, et al. Measurement of short-chain carbohydrates in common Australian vegetables and fruits by highperformance liquid chromatography (HPLC). J Agric Food Chem. Jan 28 2009;57:554-565.

9. Biesiekierski JR, Rosella O, Rose R, et al. Quantification of fructans, galacto-oligosacharides and other short-chain carbohydrates in processed grains and cereals. J Hum Nutr Diet. Apr 2011;24:154-176.

10. Manichanh C, Eck A, Varela E, et al. Anal gas evacuation and colonic microbiota in patients with flatulence: effect of diet. Gut. 2014;63:401-408.

11. Azpiroz F, Hernandez C, Guyonnet D, et al. Effect of a low-flatulogenic diet in patients with flatulence and functional digestive symptoms. Neurogastroenterol Motil. Feb 19 2014;26:779-785.

12. Serra J, Azpiroz F, Malagelada J-R. Gastric distension and duodenal lipid infusion modulate intestinal gas transit and tolerance in humans. Am J Gastroenterol. 2002;97:2225-2230.

13. Serra J, Azpiroz F, Malagelada J-R. Mechanisms of intestinal gas retention in humans:impaired propulsion versus obstructed evacuation. Am J Physiol. 2001;281:G138-G143.

14. Serra J, Azpiroz F, Malagelada J-R. Intestinal gas dynamics and tolerance in humans. Gastroenterology. 1998;115:542-550.

15. Serra J, Azpiroz F, Malagelada J-R. Impaired transit and tolerance of intestinal gas in the irritable bowel syndrome. Gut. 2001;48:14-19.

16. Nilsson M, Sandberg TH, Poulsen JL, et al. Quantification and variability in colonic volume with a novel magnetic resonance imaging method. Neurogastroenterol Motil. Dec 2015;27:1755-1763.

17. Altman DG, Bland JM. Measurement in medicine: the analysis of method comparison studies. The Statistician. 1983;32:307-317. 
8

WI LEY-Neurogastroenterology \& Motility NG M

BENDEZÚ ET AL.

1 18. Marciani L, Gassed KC, Hoad CL, et al. Stimulation of colonic motil-
ty by oral PEG electrolyte bowel preparation assessed by MRI: comparison of split vs single dose. Neurogastroenterol Moil. Oct 2014;26:1426-1436.

19. Pritchard SE, Marciani L, Gassed KC, et al. Fasting and postprandial volumes of the undisturbed colon: normal values and changes in diarrhea-predominant irritable bowel syndrome measured using serial MRI. Neurogastroenterol Moil. Jan 2014;26:124-130.

20. Mego M, Accarino A, Malagelada JR, Guarner F, Azpiroz F. Accumulahive effect of food residues on intestinal gas production. Neurogastroenteral Motil. Nov 2015;27:1621-1628.

21. Azpiroz F. Intestinal gas. In: Feldman M, Friedman LS, Brand LJ, eds. Pathophysiology, Diagnosis, Management, 10th edh. Philadelphia, USA: Elsevier; 2015:242-250.

22. Christ SU, Murgatroyd PR, Gibson GR, Cummings JH. Production, metabolism, and excretion of hydrogen in the large intestine. Gastroenterology. 1992;102:1269-1277.

23. Levitt MD, Harsh P, Fetzer CA, Sheahan M, Levine AS. H2 excretion after ingestion of complex carbohydrates. Gastroenterology. Feb 1987;92:383-389.
24. Levitt MD. Production and excretion of hydrogen gas in man. N Eng J Med. 1969;281:122-127.

25. Mego M, Bendezu A, Accarino A, Malagelada JR, Azpiroz F. Intestihal gas homeostasis: disposal pathways. Neurogastroenterol Motil. 2015;27:363-369.

26. Suarez F, Furne J, Springfield J, Levitt M. Insights into human colonic physiology obtained from the study of flatus composition. Am J Physiol. May 1997;272:G1028-G1033.

27. Passos MC, Tremolaterra F, Serra J, Azpiroz F, Malagelada J-R. Impaired reflex control of intestinal gas transit in patients with abdominal bloating. Gut. 2005;54:344-348.

28. Harder H, Serra J, Azpiroz F, Malagelada J-R. Reflex control of intestinal gas dynamics and tolerance in humans. Am J Physiol. 2004;286:G89-G94.

29. Serra J, Salvioli B, Azpiroz F, Malagelada JR. Lipid-induced intestinat gas retention in the irritable bowel syndrome. Gastroenterology. 2002;123:700-706.

30. Lassen RB, Bond JH, Levitt MD. The role of intestinal gas in functional abdominal pain. N Enol J Med. Oct 11 1975;293:524-526.

31. Furne JK, Levitt MD. Factors influencing frequency of flatus emission by healthy subjects. Dig Dis Sci. Aug 1996;41:1631-1635. 


\section{Graphical Abstract}

The contents of this page will be used as part of the graphical abstract of html only. It will not be published as part of main.
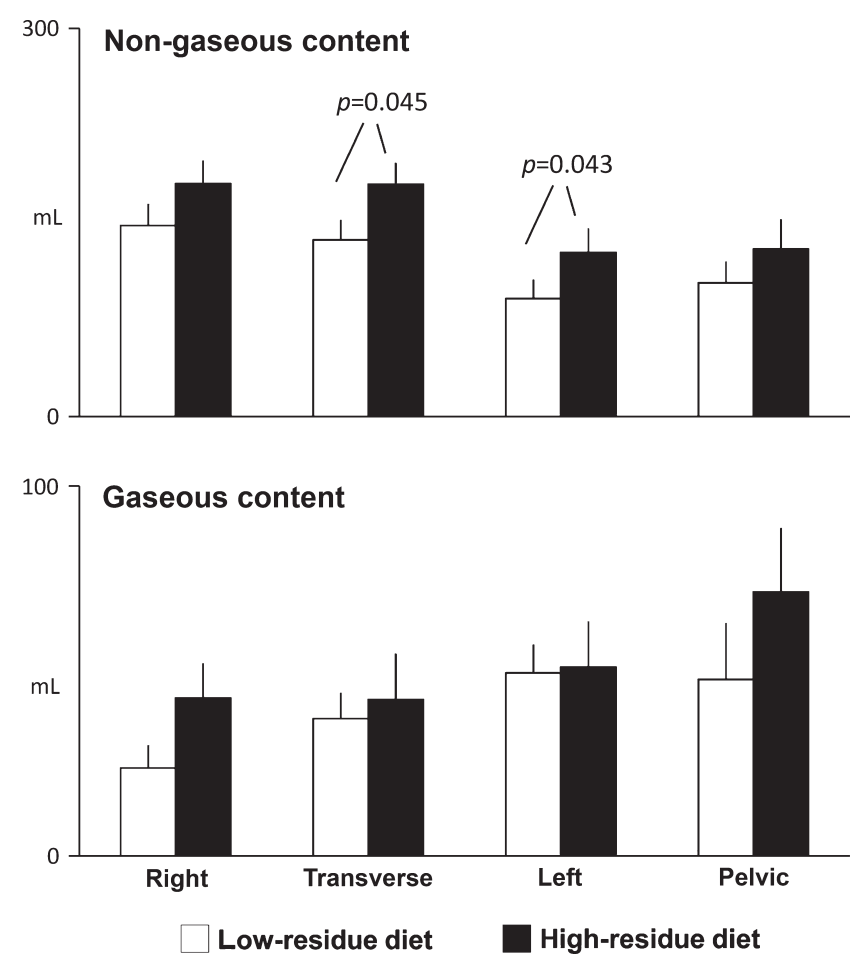

8 Ingestion of unabsorbable residues and defecation produce profound changes in colonic content. The rapid turnover of colonic biomass (about $1 / 3$ daily) indicates a high adaptation potential of microbiota to the intraluminal environment. 